\title{
BIO-INSPIRED ACOUSTIC SENSING WITH ULTRA-HIGH ASPECT-RATIO, MICROSPHERE-TIPPED PDMS MICROPILLARS
}

Jungwook Paek and Jaeyoun Kim

Department of Electrical and Computer Engineering, Iowa State University, Ames, Iowa, USA

\begin{abstract}
The cricket's cercal acoustic receptors with their long and thin filiform hairs produce excellent performance in acoustic sensing, attracting numerous attempts to mimic them. However, their replication requires high aspect-ratio micropillars, which is a great difficulty when attempted with conventional micro-fabrication techniques and PDMS. Here, we present a new technique based on the direct drawing technique incorporated with the in situ heating, which enables the realization of PDMS micropillars with unprecedented aspect-ratios $(40 \sim 112)$. Our scheme also allows self-aligned capping of the micropillars with microspheres. To validate the utility of the microsphere-tipped micropillar (MSMP), we transform it into all-optically interrogated acoustic sensors inspired by the filiform hairs.
\end{abstract}

\section{INTRODUCTION}

Nature is replete with tiny wind and sound sensors such as the cricket's cercal acoustic receptors shown in Fig.1 [1]. Using their thin and long filiform hairs, the receptors produce excellent performance in acoustic sensing, attracting numerous attempts to mimic them [2]. However, their replication requires high aspect-ratio micropillars, which is a great difficulty when attempted with conventional, replica-molding-based fabrication techniques and soft materials such as PDMS [3]. Here, we present a new technique, which enables the realization of PDMS micropillars with unprecedented aspect-ratios $(40 \sim 112)$, through a combined utilization of direct drawing and in situ thermal hardening [4]. Our technique also allows automatic integration of multi-functional microspheres at the tips of the micropillars in a self-aligned fashion.

To validate the utility of the microsphere-tipped micropillar (MSMP), we configure it into all-optically interrogated airflow sensors inspired by the filiform hairs.

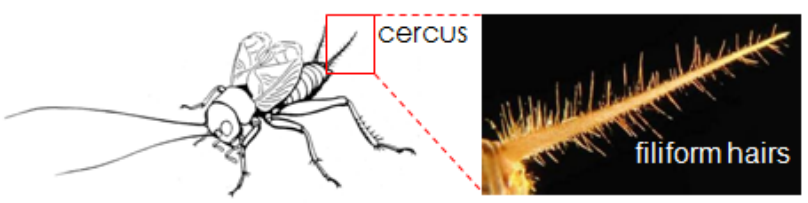

Figure 1: The cricket's cercus hosts a large number of filiform hairs as shown in the inset (from Ref. 1). They deform in response to airflows and generate signals for wind and sound reception.

\section{MICROPILLAR FABRICATION}

Steps for the PDMS MSMPs fabrication are depicted in Fig. 2. We prepared an array of $58 \mu \mathrm{m}$-diameter Ag-coated hollow glass microspheres on a piece of double-stick tape as the drawing probe (Fig. 2a). Then we attached the array to a micromanipulator. Also, we spin-coated a thin layer of PDMS and pre-baked it on a hot plate at $100^{\circ} \mathrm{C}$ (Fig. 2b). During the pre-bake, we lowered the microsphere array towards the PDMS thin film until the separation was reduced to $50 \mu \mathrm{m}$. After the pre-bake, we further lowered the array so that it can physically contact the PDMS film (Fig. 2c). After $40 \mathrm{sec}$, we lifted the micromanipulator, drawing PDMS micropillars (Fig. 2d). The completed PDMS micropillars were then post-baked at $100^{\circ} \mathrm{C}$ for 2 hours and cured at room temperature for 24 hours.
Then we detached the microspheres from the tape, leading to the realization of the PDMS MSMPs (Fig. 2e). Fig. 3 shows fabricated high aspect-ratio MSMPs.

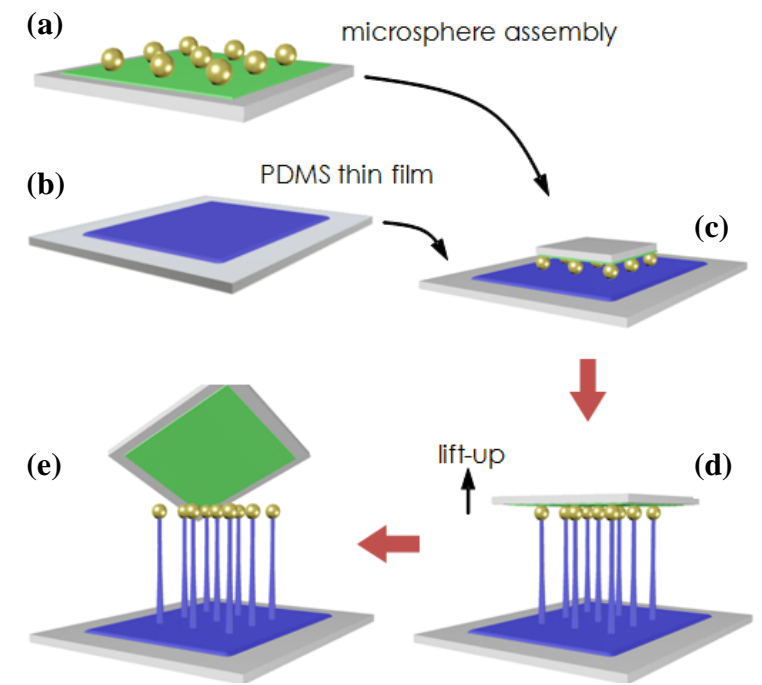

Figure 2:Fabrication flow (a) microsphere assembly (b) PDMS thin film (c) Contact between the microsphere array and PDMS thin film (d) Directly drawing PDMS micropillars (e) Detaching MSMPs from the tape (a)

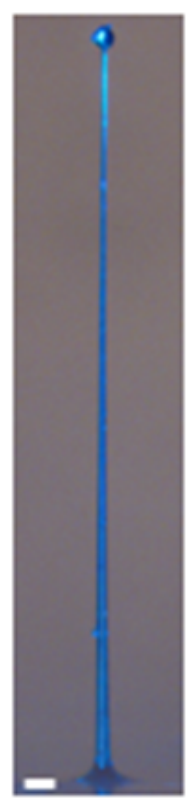

(b)

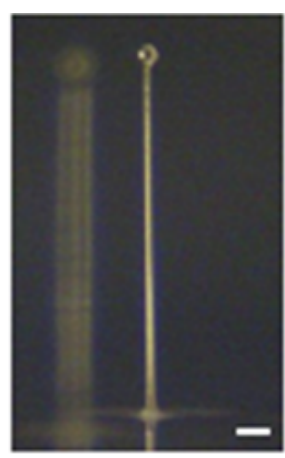

(c)

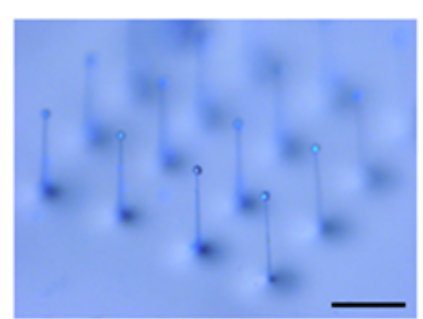

Figure 3: Optical micrographs of (a) PDMS micropillar with an aspect-ratio of 112 (scale bar: $100 \mu \mathrm{m})(\mathrm{b})$ PDMS micropillar with an aspect ratio of 84 (Scale bar: $100 \mu \mathrm{m})(\mathrm{c}) 4 \times 4$ array comprising PDMS micropillars with an average aspect-ratio of 42 (Scale bar: $700 \mu \mathrm{m})$
Solid-State Sensors, Actuators and Microsystems Workshop Hilton Head Island, South Carolina, June 8-12, 2014 


\section{BIO-INSPIRED ACOUSTIC SENSING}

To demonstrate acoustic sensing capabilities of the PDMS MSMPs, we turn three MSMPs in Table 1 into mirror-coupled flexible waveguides incorporated with optical read-out interfaces. The micropillar functions as a flexible waveguide which deforms under airflows, and the microsphere as a self-aligned reflector which reports the micropillar's level of deformation as shown in Fig.4a. Fig.4b, c shows the MSMP-A's deformation in the absence and presence of airflow, respectively. In the former, the MSMP stays straight, producing maximal reflection. Upon contact with a constant airflow, it bends due to the fluidic drag force, which decreases the reflection. The change in the reflectance is also plotted in Fig. 5 for all three MSMPs as a function of the airflow rate. The results indicate that the reflectance is a good measure of the airflow rate.

Table 1: Characteristics of the PDMS micropillars under test

\begin{tabular}{|c|c|c|c|}
\hline & Height (um) & $\begin{array}{c}\text { Ave. diameter (um) } \\
\text { (top, middle, base) }\end{array}$ & Aspect-ratio \\
\hline MSMP-A & 800 & 19.8 & 40.4 \\
\hline MSMP-B & 1600 & 22 & 72.7 \\
\hline MSMP-C & 2000 & 23 & 86.9 \\
\hline
\end{tabular}

(a)

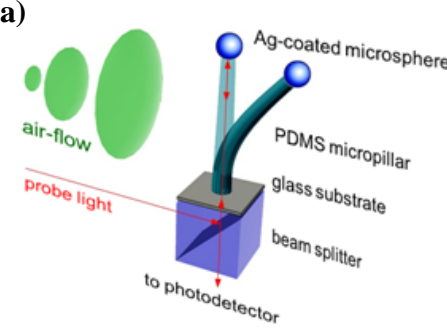

(b)

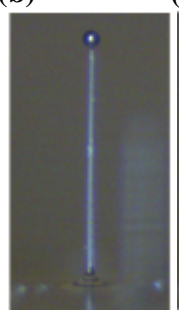

(c)

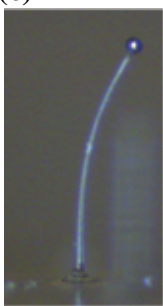

Figure 4: (a) The optical read-out setup for MSMP-based airflow sensing $(b)$ and $(c) M S M P$-A's bending in response to the absence and presence of airflow, respectively

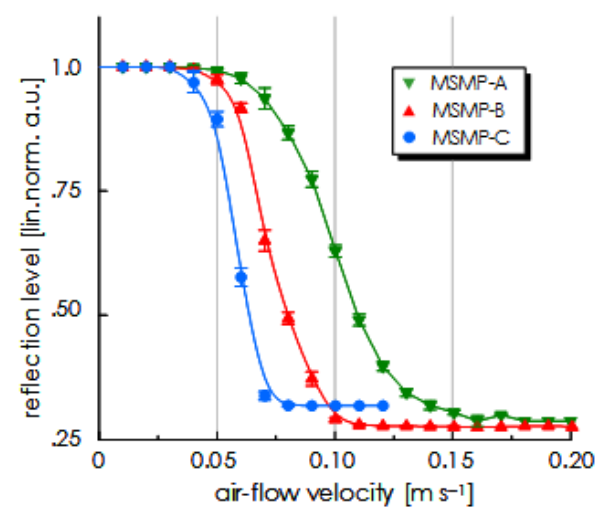

Figure 5: The change in the micropillar's reflection level as a function of the airflow velocity. The error bars represent the corresponding standard deviations.

We also investigated the utility of the MSMPs for sensing time-varying airflows, i.e., sound-waves. Fig. 6a shows the optical reflection from MSMP-C responding to a sound-wave at $90 \mathrm{~Hz}$ with two identical dips per one temporal period. The shades in the plot represent one temporal period. The resulting generation of higher harmonics is evident in the Fourier transform of the $90 \mathrm{~Hz}$ response as shown in Fig. 6b, providing accurate information on the frequency of the sound-wave. MSMP-A and B generated similar reflection pattern. Fig. 6c shows how modulation-depth of the reflection output changes as a function of the excitation frequency over $60 \sim 200 \mathrm{~Hz}$, showing $102 \mathrm{~Hz}$ and $93 \mathrm{~Hz}$ as the peak frequency of MSMP-B and C, respectively. Resonance at this low frequency range is difficult to obtain with cantilevers made of hard materials.

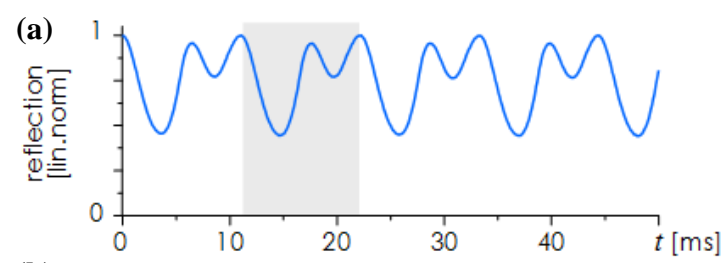

(b)
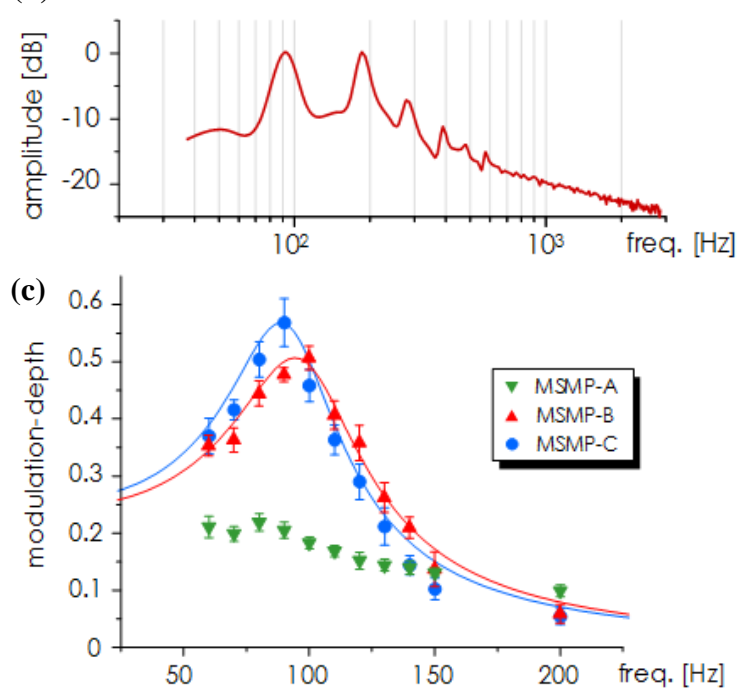

Figure 6: (a) Temporally changing reflections from MSMP-C in response to sound waves at $90 \mathrm{~Hz}$ (b) The amplitude portion of the Fourier transform of the $90 \mathrm{~Hz}$ output (c) The modulation-depth of the temporal responses from the three MSMPs as a function of the excitation frequency

\section{CONCLUSION}

We present a new soft-lithography technique which not only enables the realization of high aspect-ratio PDMS micropillars but also allows integration of multi-functional microspheres in a self-aligned fashion. Bio-inspired acoustic sensors we configured based on the micropillars exhibited high sensitivity at resonance frequencies close to $100 \mathrm{~Hz}$.

\section{REFERENCES}

[1] Miller, J. P., Krueger, S., Heys, J. J. and Gedeon, T.,"Quantitative Characterization of the Filiform Mechanosensory Hair Array on the Cricket Cercus",PLoS ONE6, e27873 (2011)

[2] Dijkstra, M. et al, "Artificial sensory hairs based on the flow sensitive receptor hairs of crickets",J. Micromech. Microeng. 15, S132 (2005).

[3] Große, S., Schröder, W. and Brücker, C., "Nano-newton drag sensor based on flexible micro-pillars",Meas. Sci. Technol. 17, 2689 (2006).

[4] Paek, J. and Kim, J., "Microsphere-assisted fabrication of high aspect-ratio elastomeric micropillars and waveguides", Nature Communications, 5, 3324 (2014). 